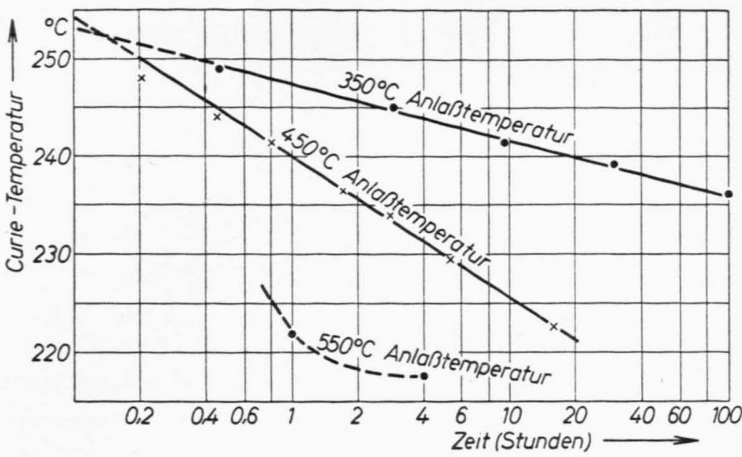

Abb. 1. Curie-Punktsverschiebung in Abhängigkeit von der Temperdauer.

mender Temperdauer in Richtung auf einen später auftretenden dritten Curie-Punkt bei $210^{\circ} \mathrm{C}$, den des Zementits $\left(\mathrm{Fe}_{3} \mathrm{C}\right)$. Diese CurIE-Punktsverschiebung ist in den thermomagnetischen Untersuchungen, die Crangle, Sucksmith ${ }^{3}$ und Apajev ${ }^{4}$ im gleichen Temperaturbereich an vergleichbarem Probenmaterial durchgeführt haben, nicht gefunden worden.

5 K. H. JACK, J. Iron Steel Inst. (London) 169, 26 [1951].
Der wandernde CuRIE-Punkt ist einer verspannten zementitähnlichen Phase zuzuschreiben, die von $\mathrm{J}_{\mathrm{ACK}}{ }^{5}$ schon röntgenographisch nachgewiesen wurde. Die Verspannung dieser Phase heilt beim Tempern aus, was in einer Wanderung des CuRIE-Punktes in Richtung auf den Curie-Punkt des unverspannten Zementits zum Ausdruck kommt.

Belous und Cherepin ${ }^{6}$ haben durch schrittweise Erhöhung des Kaltverformungsgrades eines Zementit enthaltenden Stahles eine CurIE-Punktsverschiebung in umgekehrter Richtung von $210^{\circ} \mathrm{C}$ auf $250^{\circ} \mathrm{C}$ gefunden.

Aus unseren Meßergebnissen kann gefolgert werden, $\mathrm{da} ß$ sich während der 3 . Temperstufe unter spontanem Zerfall des $\varepsilon$-Eisencarbids sowohl eine verspannte zementitähnliche Zwischenphase als auch $\chi$-Carbid $\left(\sim \mathrm{Fe}_{20} \mathrm{C}_{9}\right)$ und nach ausreichend langer Temperdauer aus beiden Phasen schließlich Zementit bildet. Über Einzelheiten der Bildungs- und Zerfallsvorgänge wird an anderer Stelle an Hand der vorliegenden Ergebnisse noch ausführlich berichtet.

Herrn Prof. Dr. H. Lange möchten wir für die stete Förderung unserer Arbeit recht herzlich danken.

${ }^{6}$ M. V. Belous u. V. T. Cherepin, Fiz. Metal. i Metalloved. (engl. Ubers.) 14, 140 [1962].

\section{Gasadsorptionsverhalten, Hall-Effekt und Struktur von Wismutschichten}

\section{Von L. Fritsche und H. Seufert}

Institut für theoretische Physik der Technischen Hochschule Karlsruhe

(Z. Naturforschg. 18 a, 1013-1015 [1963] ; eingeg. am 2. August 1963)

Die elektrischen Eigenschaften dünner Wismutschichten sind wegen der besonderen Stellung des Wismuts unter den Metallen häufig untersucht worden. Insbesondere über das Verhalten der spezifischen Leitfähigkeit und der Elektronen-Austrittsarbeit bei der Adsorption von Gasen liegen umfangreiche Messungen von SuHRmann und Mitarbeitern vor (s. Suhrmann und Keune ${ }^{1}$, Suhrmann und Wedler ${ }^{2}$, Suhrmann, Wedler und DierK $^{3}$ ). Die Deutung dieser Messungen, speziell die aus ihnen entwickelten Vorstellungen über den Bindungscharakter des Sauerstoffs bei der Adsorption sind nicht zwingend und werden z. Tl. bezweifelt (s. SuHrMANN und $\mathrm{W}_{\text {EDLer }}{ }^{2}$, Diskussionsbemerkung SachtLer). Die von Suhrmann untersuchten Schichten zeigen ferner einen negativen Temperaturkoeffizienten des Widerstands, aus dem auf Halbleitereigenschaften geschlossen wird. Diese Vermutung wird gestützt durch Messungen

1 R. Suhrmann u. H. Keune, Z. Elektrochem. 60, 898 [1956].

2 R. Suhrmann u. G.Wedler, Z. Elektrochem. 63, 748 [1959].

3 R. Suhrmann, G. Wedler u. E.-A. Dierk, Z. Phys. Chem. 18, 256 [1958]. von Colombani und Mitarbeitern (s. z. B. Colombani und $\mathrm{HUET}^{4}$ ), der für Schichten, die bei Zimmertemperatur aufgedampft werden, stets negative HALL-Konstanten findet. Im Gegensatz hierzu stehen Messungen von Buckel und Hilsch (s. z. B. Bucket ${ }^{5}$ ) an Schichten, die bei $4{ }^{\circ} \mathrm{K}$ kondensiert werden. Sie liefern auch bei Zimmertemperatur positive Werte der HALL-Konstanten, haben also n-Leitungscharakter. Colombani zeigt jedoch, daß Schichten seiner Herstellungsbedingungen - Wismut zwischen zwei amorphen $\mathrm{Bi}_{2} \mathrm{O}_{3}$-Filmen eingebettet - durch Erhitzen über den Schmelzpunkt ebenfalls n-leitend werden. Dennoch kann der Temperaturkoeffizient des Widerstands negativ bleiben.

Eine Klärung dieses sehr uneinheitlich scheinenden Verhaltens des Wismuts kann nur von einer genauen Diskussion der Schichtstruktur erwartet werden, die sich bei den verschiedenen Herstellungsbedingungen ergibt.

Die bei $4{ }^{\circ} \mathrm{K}$ kondensierten, zunächst amorphen Wismutfilme unterscheiden sich oberhalb ihrer Kristallisationstemperatur von $20^{\circ} \mathrm{K}$ qualitativ nicht vom polykristallinen Kompaktmaterial. Es ist daher zu vermuten, daß Schichten, die oberhalb $20^{\circ} \mathrm{K}$ unmittelbar als polykristalliner Niederschlag entstehen, noch eine zusätzliche Überstruktur besitzen, die ihre besonderen

4 A. Colombani u. P. Huet, C. R. Acad. Sci., Paris 244, 755 [1957].

5 W. Buckes, Beitrag in: Structure and Properties of Thin Films, John Wiley \& Sons, Inc., New York u. London 1959, S. 53 . 
elektrischen Leitungseigenschaften erklärt und auch bei der Deutung des Gasadsorptionsverhaltens zu berücksichtigen ist.

Zur Untersuchung dieses Zusammenhangs haben wir Wismutfilme bei einer Unterlagentemperatur von $300{ }^{\circ} \mathrm{K}$ im Ultrahochvakuum mit einer Massenbelegungsdichte von einigen $10^{-5} \mathrm{~g} \cdot \mathrm{cm}^{-2}$ hergestellt. Auf Glas als Unterlage bilden solche Schichten bei Normaltemperatur keinen zusammenhängenden Film. Deshalb verwenden Suhrmann und Mitarbeiter $90{ }^{\circ} \mathrm{K}$ als Kondensationstemperatur. Erfahrungsgemäß werden aber beim Erhitzen der Verdampfungsspiralen bzw. -Öfen größere Mengen $\mathrm{CO}$ und $\mathrm{CO}_{2}$ frei, die bei dieser Temperatur zusammen mit dem Wismut kondensieren und damit von vornherein die Reinheit der Versuchsbedingungen in Frage stellen. Wir verwenden daher $\mathrm{Bi}_{2} \mathrm{O}_{3}$ als Unterlage, das wegen seiner Adhäsionswirkung auf die kondensierenden Wismutatome auch bei $300{ }^{\circ} \mathrm{K}$ eine Inselbildung verhindert. Die Schichten werden nach dem Destillationsverfahren von $\mathrm{ORT}^{6}$ aufgedampft. Die etwas abgewandelte Anordnung hierzu besteht aus zwei Teilen (Abb.1). Der von außen magnetisch bewegbare Verdampfer wird induktiv erhitzt und bei ca. $1200{ }^{\circ} \mathrm{C}$ gründlich entgast. Danach wird eine kleine

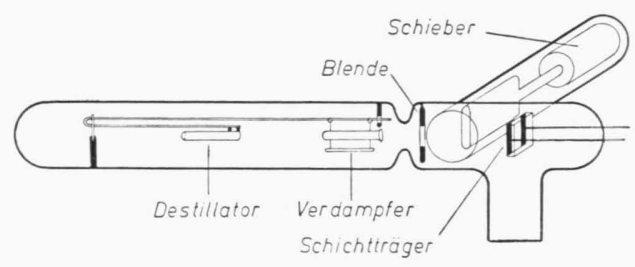

Abb. 1. Meßzelle.

Menge Wismut (spektralrein, Hersteller: J o hns on \& M a t the y Ltd., London) in den Verdampfer überdestilliert, wobei sich die Öffnungen von Verdampfer und Destillator gegenüberstehen. Der Restgasdruck beträgt bei diesem Prozeß ca. $1 \cdot 10^{-8}$ Torr. Das Destillat ist $\mathrm{i}$. allg. ausreichend entgast, anderenfalls erzielt man durch wiederholtes Hin- und Rückdampfen Verbesserung. Bei der Herstellung der Schicht nimmt der Verdampfer die dargestellte Lage ein. Der Schichtträger hat einen Abstand von ca. $50 \mathrm{~mm}$. Der Restgasdruck beträgt bei einer Bedampfungsgeschwindigkeit von $1 \AA \cdot \mathrm{s}^{-1}$ während der Bedampfungszeit von $3-5 \mathrm{~min}$ ca. $2 \cdot 10^{-9}$ Torr. (Nach der Restgasanalyse mit dem Omegatron hat der Sauerstoffpartialdruck dabei höchstens den Wert von $1 \cdot 10^{-10}$ Torr.) Der Schichtträger aus Duranglas ist mit Goldkontakten versehen und wird zuvor in einer Anordnung nach Gillham und Preston ${ }^{7}$ mit einem $150 \AA$ dicken $\mathrm{Bi}_{2} \mathrm{O}_{3}$-Film bis zur Mitte der Kontaktstreifen bedeckt. Die Meßzelle ist an eine UHV. Apparatur - bestehend aus einer Öldiffusionspumpe

6 W. ORT, Z. Phys. 163, 230 [1961].

7 E. J. Gillham u. J. S. Preston, Chem. Age 1952, S. 67.

8 M. N. Markov u. J. S. Lindstrom, Soviet Phys.-Solid State 1, 749 [1959].

9 R. Suhrmann u. H. Schnackenberg, Z. Phys. 119, 287 [1942]. und einer $8 l \cdot \mathrm{s}^{-1}$-Vac-Ion-Pumpe - angeschmolzen. Für Adsorptionsversuche werden die Gase in spektralreiner Form über ein Ventil (System Granville$\mathrm{P}$ hilli p s) aus Vorratsflaschen eingelassen.

Die Untersuchungen ergeben folgendes Verhalten der Schichten:

1. Die spezifische Leitfähigkeit der Schichten von $300 \AA$ Dicke ist um den Faktor 7 kleiner als die des Kompaktmaterials (vgl. hierzu Markov und Lindstrom ${ }^{8}$ ). Nach dem Aufdampfen erniedrigt sich der Schichtwiderstand nach einem hyperbolischen Zeitgesetz (vgl. hierzu Suhrmann und Schnackenberg ${ }^{9}$ ). Tempern bis $\mathrm{zu}$ $120{ }^{\circ} \mathrm{C}$ beschleunigt diesen Prozeß. Der Temperaturkoeffizient des Widerstands ist negativ, nach Messungen von Wolber ${ }^{10}$ ebenso das Vorzeichen der HallKonstanten.

2. Oberhalb der Schmelztemperatur fällt der Widerstand steil ab. Nach dem Wiedererstarren hat die HALLKonstante ihr Vorzeichen gewechselt ${ }^{10}$, der Temperaturkoeffizient nur in manchen Fällen (vgl. hierzu z. B. Colombani ${ }^{11}$ ).

3. Ungetemperte Schichten von ca. $100 \AA$ Dicke zeigen bei Drucken von $1 \cdot 10^{-8}$ Torr eingelassenen Sauerstoffs eine meßbare Widerstandserniedrigung, die bei $1 \cdot 10^{-5}$ Torr auf $1 \%$ maximal angewachsen ist. Bei größeren Zugabedrucken tritt eine erhebliche Widerstandserhöhung auf (vgl. Suhrmann und Keune ${ }^{1}$ ). Bis $120{ }^{\circ} \mathrm{C}$ getemperte Schichten liefern im ganzen Druckbereich nur Widerstandserhöhung bei zunehmendem Betrag der HALL-Konstanten ${ }^{10}$.

4. Durch Zugabe von Wasserstoff kann die Widerstandszunahme einer mit Sauerstoff belegten, ungetemperten Schicht rückgängig gemacht und der Anfangswiderstand sogar unterschritten werden (Abb. 2). Der Betrag der HaLL-Konstanten nimmt dabei $\mathrm{ab}^{10}$. Bei einer bis $120^{\circ} \mathrm{C}$ getemperten, anschließend mit Sauerstoff belegten Schicht kann - unter sonst gleichen Versuchsbedingungen - die Widerstandszunahme nur z. Tl. rückgängig gemacht werden (Abb. 3).

5. Ohne Vorbelegung mit Sauerstoff bewirkt Wasserstoffzugabe bis zu $10^{-3}$ Torr eine Widerstandserniedrigung von nur $3 \% 0$.

6. Ebenso erzeugt Wasserdampf bis zu $3 \cdot 10^{-3}$ Torr an unbelegten, ungetemperten Filmen eine Widerstandsabnahme von nur $2 \%$.

Diese Ergebnisse legen folgende Deutung nahe:

1. Die Schichten bilden ein poröses Kristall-Konglomerat mit merklichen Übergangswiderständen an den Kontaktstellen der Körner (vgl. hierzu EHRLich ${ }^{12}$, der von ähnlichen Überlegungen ausgeht). Durch Altern und Tempern werden diese Übergangswiderstände erniedrigt, außerdem durch die gegenseitige thermische Ausdehnung der Körner bei Temperaturerhöhung, da der Schichtträger einen kleineren thermischen Ausdeh-

10 W. Wolber, Diplomarbeit, Karlsruhe 1963.

11 A. Colombani u. P. Huet, Beitrag in: Structure and Properties of Thin Films, John Wiley \& Sons, Inc., New York und London 1959, S. 253.

12 G. Ehrlich, J. Chem. Phys. 35, 2165 [1961]. 


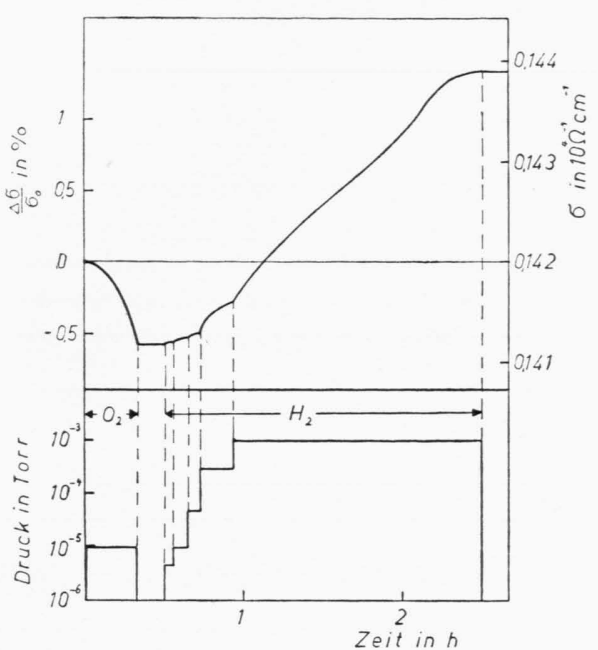

Abb. 2. Leitfähigkeitsänderung bei Adsorption von Sauerstoff und anschließend Wasserstoff an einer ungetemperten Schicht.

nungskoeffizienten hat. Daraus folgt ein negativer Temperaturkoeffizient des Widerstands.

Die Schichten wachsen partiell orientiert, mit der trigonalen Achse des $\mathrm{Bi}$ vorwiegend senkrecht zur amorphen Unterlage auf (s. hierzu LANE ${ }^{13}$, Bound und Richards ${ }^{14}$ ). Bei der Hall-Effektmessung ist das Magnetfeld parallel zu dieser Vorzugsrichtung. WismutEinkristalle zeigen bei einer solchen Anordnung ebenfalls Überschuß-p-Leitung (s. hierzu Gitsu und IvaNov $\left.{ }^{15}\right)$.

2. Beim Erhitzen über den Schmelzpunkt geht das Wismut in die sehr viel besser leitende amorphe Phase über, wie sie bei den Experimenten von Buckel und Hilsch (vgl. Buckel ${ }^{5}$ ) unterhalb von $4{ }^{\circ} \mathrm{K}$ in eingefrorener Form auftritt. Nach dem Wiedererstarren ist die Vorzugsorientierung der Kristallite zerstört. Man mißt

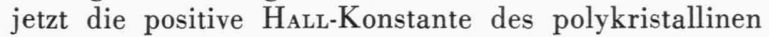
Wismuts.

3. Eingelassener Sauerstoff wird bei jedem Druck auf der Oberfläche der Wismutkriställchen chemisorbiert. Dabei bilden die Mikrospalte an Kontaktübergängen besonders reaktive Stellen. Nach Messungen von $\mathrm{ORT}^{6}$ bewirkt aber ein Film chemisorbierten Sauerstoffs in solchen Spalten eine Erniedrigung des Kontaktwiderstandes. Sind die Spalten mit Sauerstoff gesättigt, kann keine weitere Erniedrigung mehr erzielt werden. Bei größeren Drucken des Sauerstoffs erfolgt die Chemisorption nur mehr an den freien Oberflächen, insbesondere an den festen Kontaktbrücken (vgl. EHR${ }_{\text {LICH }}{ }^{12}$ ). Dadurch erhöht sich der Widerstand, da durch

13 C. T. Lane, Phys. Rev. 48, 193 [1935].

14 M. Bound u. D. A. Richards, Proc. Roy. Soc., Lond. 51, 256 [1939]. die Chemisorption die Zahl der Leitungselektronen abnimmt.

Bei getemperten Schichten sind die Mikrospalte eliminiert und können zum Gasadsorptionsverhalten nicht mehr beitragen.

4. Wegen der reaktiven Eigenschaften der Mikrospalte wird nachträglich eingelassener Wasserstoff vorzugsweise dort angreifen und die Bindung des Sauerstoffs lockern oder ganz aufheben (Abb.2). Es wäre denkbar, daß eine sich dort bildende Wasserhaut eine besonders wirksame Kontaktbrücke herstellt. Bei größeren Zugabedrucken des Wasserstoffs kommen wieder die freien Oberflächen ins Spiel. Lockern und Entfernen des Sauerstoffs durch Reaktion mit dem Wasserstoff erhöht die Zahl der Leitungselektronen, und der Betrag der (negativen) HaLL-Konstanten nimmt ab ${ }^{10}$. Offenbar können die freien Oberflächen aber nicht vollständig reduziert werden, denn bei getemperten Schichten verbleibt ein Resteffekt des Sauerstoffs (Abb. 3).

5. Nach Messungen von Sieverts und Krumbhaar ${ }^{16}$ tritt bei Wasserstoff keine Chemisorption an reinen Wismutoberflächen auf. Entsprechend ist die Widerstandsänderung klein. Das gleiche gilt auch für Wasserdampf.

Eine ausführliche Beschreibung und Diskussion weiterer Untersuchungen wird an anderer Stelle gegeben werden.

Wir benutzten zahlreiche Instrumente und sonstige Hilfsmittel, die Herrn Professor Wolf von der Deutschen Forschungsgemeinschaft zur Verfügung gestellt waren.

15 D. V. Girsu u. G. A. Ivanov, Soviet Phys.-Solid State 2, 1323 [1961].

16 A. Sieverts u. W. Krumbhaar, Ber. dtsch. chem. Ges. 43, 893 [1910]. 